\title{
CORRIGENDUM
}

\section{Schizophrenia genes, gene expression, and neuropathology: on the matter of their convergence}

PJ Harrison and DR Weinberger

Molecular Psychiatry (2005) 10, 420. doi:10.1038/sj.mp.4001630

Correction to: Molecular Psychiatry (2005) 10, 40-68. doi:10.1038/sj.mp.4001558

Following publication of the above paper, the author has identified an error on the sixth page. In the lefthand column, 10 lines from the bottom, the sentence should simply read: 'COMT has significantly lower enzyme activity than net-COMT.' 